\title{
Sirolimus Therapy and Follow-up in a Patient with Severe Congenital Hyperinsulinism Following Subtotal Pancreatectomy
}

\author{
(D) Qiong Chen ${ }^{1}$, (D) Yongxing Chen ${ }^{1}$, (D) Xiaohong Wang1, (D) Haihua Yang1, (D) Yingxian Zhang1, (D) Xiaojing Liu1, (D) Yun Yan², \\ (D) Haiyan Wei1 \\ ${ }^{1}$ Henan Children's Hospital (Children's hospital affiliated to Zhengzhou University), Department of Endocrinology and Metabolism, Genetics, \\ Zhengzhou, China \\ 2University of Missouri-Kansas City, Children's Mercy Hospital, Department of Endocrinology and Diabetes, Missouri, USA
}

\begin{abstract}
What is already known on this topic?
Congenital hyperinsulinism (CHI) is the most common cause of persistent hypoglycemia in neonates and infants. Sirolimus may be an effective treatment option in patients with $\mathrm{CHI}$ resistant to traditional medical therapy or failure of subtotal pancreatectomy, but experience is limited.
\end{abstract}

\section{What this study adds?}

This article further revealed the safety and efficacy of sirolimus in the very young patient with CHI, which can make us better understand the new treatment. The patient has a heterozygous ABCC8 mutation. It's also the first Chinese CHI patient with heterozygous ABCC8 mutation who used sirolimus.

\begin{abstract}
Congenital hyperinsulinism (CHI) is the most common cause of severe, persistent hypoglycemia in neonates and infants. If the patient does not respond to medical treatment the currently available treatment is subtotal pancreatectomy, but some patients still experience severe hypoglycemia after surgery. Sirolimus, a mammalian target of rapamycin inhibitor has recently been reported to be effective in the treatment of insulinoma and CHI patients. Here we report a patient with $\mathrm{CHI}$ who had prolonged hypoglycemia after subtotal pancreatectomy. The patient had a heterozygous mutation in ABCC8 but was unresponsive to an optimal dose of diazoxide (15 mg/ $\mathrm{kg} /$ day) and octreotide (30 $\mathrm{gg} / \mathrm{kg} /$ day). The patient subsequently had subtotal pancreatectomy but severe and persistent hypoglycemia continued post-operatively. Sirolimus was commenced. There was a remarkable improvement in glycemic control without major adverse events, although he required a small dose of octreotide to maintain euglycemia. Sirolimus therapy was discontinued when the patient was 15 months old. At the time of this report, at an age of three years and eight months, the patient continues to maintain good glycemic control. This report suggests that sirolimus may be an effective treatment option in patients with CHI resistant to established medical therapy or failure of subtotal pancreatectomy. However, the long-term safety requires study in larger groups of very young patients.
\end{abstract} Keywords: Congenital hyperinsulinism, hypoglycemia, mTOR, sirolimus, ABCC8

\section{Introduction}

Congenital hyperinsulinism $(\mathrm{CHI})$, the major cause of persistent hypoglycemia in neonates and infants, is characterized by inappropriate insulin secretion from pancreatic beta cells in the presence of low blood glucose levels (1). Prompt and early management of these patients is very important for neurological prognosis $(1,2)$. The incidence of $\mathrm{CHI}$ in the general population is estimated at 1/30,000-1/50,000 live births $(3,4)$. Two major histologic subtypes have been described: diffuse (60-70\% of patients) and focal (30-40\% of patients) (5). Mutations in $A B C C 8$ and KCNJ11 cause severe $\mathrm{CHI}$ that is unresponsive to medical treatment with diazoxide and octreotide (1). The current
Address for Correspondence: Haiyan Wei MD, Henan Children's Hospital (Children's hospital affiliated to Zhengzhou University), Department of Endocrinology and Metabolism, Genetics, Zhengzhou, China Phone: +8613838521183 E-mail: haiyanwei2009@163.com ORCID: orcid.org/0000-0003-1044-6594

${ }^{\circ}$ Copyright 2021 by Turkish Pediatric Endocrinology and Diabetes Society

The Journal of Clinical Research in Pediatric Endocrinology published by Galenos Publishing House.
Conflict of interest: None declared Received: 20.02.2020 Accepted: 26.04 .2020 
treatment for patients is a subtotal pancreatectomy $(5,6)$. However, despite surgery, $40-59 \%$ of operated patients continue to experience severe and persistent hypoglycemia for months, or even years (7), and nearly $100 \%$ will develop diabetes mellitus within 11 years of surgery (8). Therefore, medical therapeutic alternatives should be considered with the aim of reducing insulin secretion and thereby preventing neurologic consequences. Constitutive activation of the mammalian target of rapamycin (mTOR) pathway has been postulated as a mechanism for hyperinsulinism and $\beta$-cell hyperplasia in diffuse $\mathrm{CHI}$ (9). Recent advances have shown the effectiveness of sirolimus, an mTOR inhibitor, in infants with severe diffuse $\mathrm{CHI}$ that had been unresponsive to medical therapy $(10,11,12,13)$, one of whom had undergone subtotal pancreatectomy (10). During follow-up, no major adverse events was observed in the patients. We report a patient with $\mathrm{CHI}$ who failed to become euglycemic after pancreatectomy. The patient was successfully treated with sirolimus without further surgical intervention.

\section{Case Report}

The patient, a male infant, was born by cesarean at the $39^{\text {th }}$ week of gestation to nonconsanguineous Chinese parents after an uneventful pregnancy. Birth weight was $3600 \mathrm{~g}$. On the first day of his life, he was found to have severe hypoglycemia when he developed lethargy and seizures. $\mathrm{He}$ required high intravenous glucose infusion rate (GIR) (13 $\mathrm{mg} / \mathrm{kg} / \mathrm{minute}$ ) to maintain normal blood glucose level. As he had persistent and severe hypoglycemia, he was transferred to our hospital for further management on postnatal day (PD) 13. The following results were obtained during an episode of hypoglycemia: glucose, 2.3 $\mathrm{mmol} / \mathrm{L}$; concomitant serum insulin, 13.52 (normal: 4.0323.46) $\mu \mathrm{IU} / \mathrm{mL}$; C-peptide 4.25 (normal: 0.3-3.73) $\mathrm{ng} /$ $\mathrm{mL}$; beta-hydroxybutyrate $<0.1 \mathrm{mmol} / \mathrm{L}$. He had normal thyroid-stimulating hormone and free T4 levels. Metabolic screening profiles in plasma and urine were non-specific. Genetic analysis subsequently confirmed a novel mutation, C.1585_1587del, in exon 10 of the $A B C C 8$ gene (Figure 1), which resulted in the deletion of glutamic acid at position 529 (p.del529E) of $A B C C 8$ protein and produced $A B C C 8$ protein with a shorter topological domain (www.ncbi.nih. gov/orffinder and www.uniprot.org). The mutations in the topological domain affected the function of the $A B C C 8$ gene (14). According to American College of Medical Genetics criteria, the mutation is of uncertain significance and should be further studied (15). His father has the same mutation, but the phenotype is normal. Magnetic resonance imaging of the patient's brain showed bilateral abnormalities of the parietal white matter. Subsequently, maximal GIR was 16 $\mathrm{mg} / \mathrm{kg} / \mathrm{min}$, administered parenterally via a central venous catheter. Diazoxide therapy was commenced on PD 14 and was gradually increased to an optimal dose of $15 \mathrm{mg} / \mathrm{kg} /$ day but with no response. On PD 20 Nifedipine was added to the therapeutic regimen, but it was discontinued after a week due to lack of response. Subcutaneous octreotide was initiated on PD 29. The octreotide dose was increased to a maximum dose (30 $\mu \mathrm{g} / \mathrm{kg} /$ day), but resulted in only a $20 \%$ reduction in total glucose requirement. On PD 55, a subtotal pancreatectomy was performed at the Children's Hospital of Fudan University, Shanghai, China. Histopathological results confirmed diffuse hyperplasia of the islet cells (Figure 2). Subcutaneous octreotide was discontinued after the surgery,

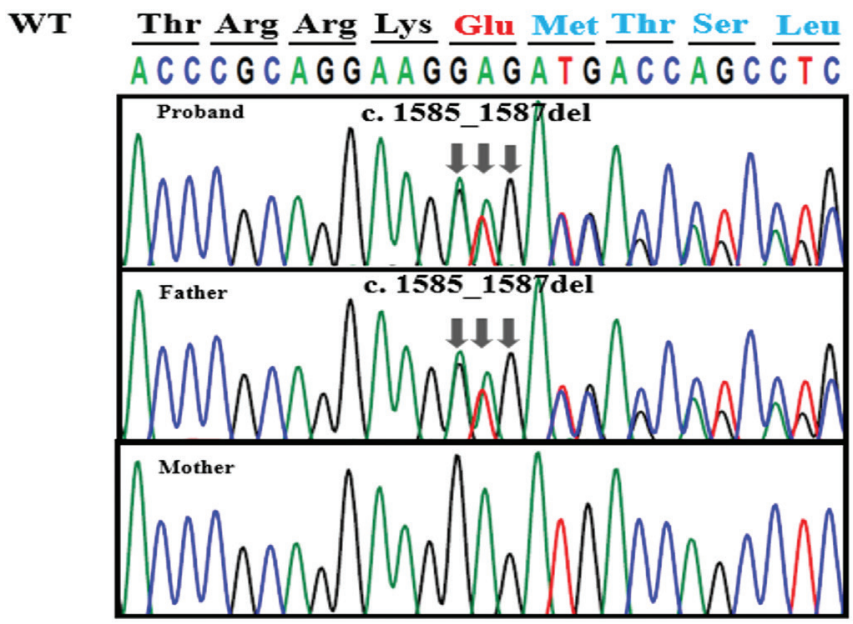

MUT ACCCGCAGGAAGAGACCAGCCTCAGG $\overline{\text { Thr }} \overline{\text { Arg }} \overline{\text { Arg }} \overline{\text { Lys }} \overline{\text { Met }} \overline{\text { Thr }} \overline{\text { Ser }} \overline{\text { Leu }} \overline{\text { Arg }}$

Figure 1. Sanger sequencing of $A B C C 8$ gene in the proband and his parents: the arrows showed the mutation site of the $A B C C 8$ gene

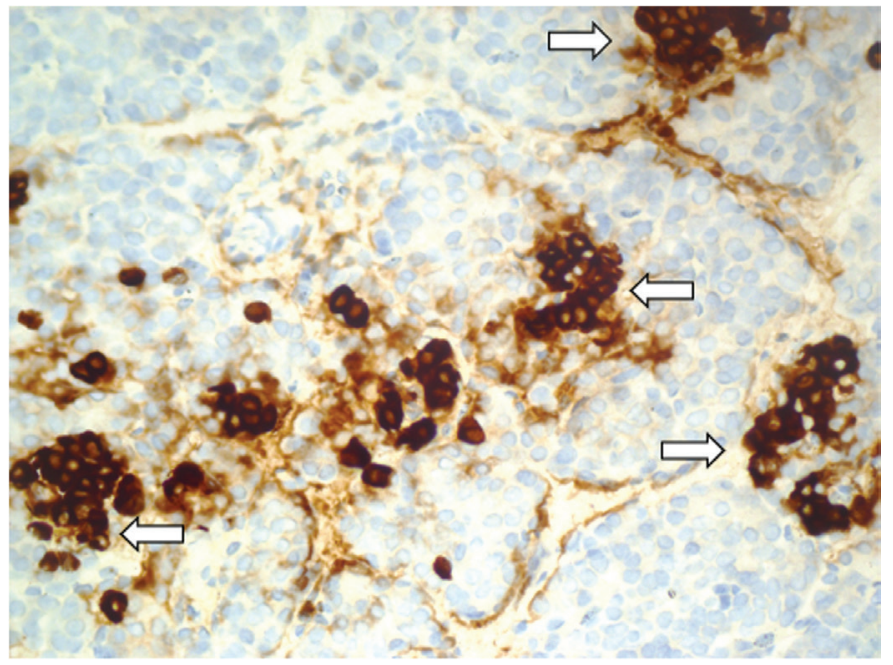

Figure 2. Histopathological result confirmed diffuse hyperplasia of the islet cells. The arrows showed hypersecretion of islet cells in islets 
but the minimum GIR remained $10 \mathrm{mg} / \mathrm{kg} / \mathrm{min}$. Octreotide subcutaneous injection was resumed with the dose of 30 $\mu \mathrm{g} / \mathrm{kg} /$ day. Over the next few weeks, there was no reduction in his glucose requirement. The total volume of nasogastric and parenteral fluids reached $190 \mathrm{~mL} / \mathrm{kg} / \mathrm{d}$ and it was also very difficult to establish a central venous line.

In view of the multiple medical problems, further surgery was being contemplated. After reviewing the risks and benefits, Sirolimus was considered as an alternative treatment option. Sirolimus treatment was begun at 4.5 months of age at a dose of $0.5 \mathrm{mg} / \mathrm{m}^{2} /$ day. The dose was gradually increased, with the goal of reaching a serum trough level of 5-15 ng/dL. The serum trough level of sirolimus was measured every 5-7 days. After 10 days of treatment with sirolimus, intravenous glucose infusion and subcutaneous octreotide were gradually tapered. Four weeks following initiation of sirolimus, stable blood glucose homeostasis was achieved without intravenous glucose infusion, and the octreotide dose was reduced from $30 \mu \mathrm{g} / \mathrm{kg} /$ day to $15 \mu \mathrm{g} / \mathrm{kg} /$ day. The patient was able to tolerate fasting for four hours while maintaining a blood glucose level $>60 \mathrm{mg} / \mathrm{dL}$ prior to discharge as recorded by continuous glucose monitoring. The patient was followed regularly for assessment of glycemic control and measurement of serum sirolimus levels. Sirolimus was discontinued at 15 months of age. The maximum dose of sirolimus used was $3.2 \mathrm{mg} / \mathrm{m}^{2} /$ day.

The patient had good glycemic control after cessation of sirolimus. However, at times of poor appetite, there was still a requirement for low dose octreotide $(2 \mu \mathrm{g} / \mathrm{kg} /$ day) to control blood glucose. Complete blood count, serum lipid profile, and renal and liver function have been monitored regularly and no significant side effects were observed, except for mildly elevated triglycerides at two years and three months old. At the time of writing the patient was three years and six months old. At the last visit, the patient was able to tolerate fasting for six hours according to continuous glucose monitoring. The blood glucose was 95 $\mathrm{mg} / \mathrm{dL}$ and the insulin was $4.9 \mu \mathrm{IU} / \mathrm{mL}$ at the end of the six hours fasting.

\section{Discussion}

The management of diffuse $\mathrm{CHI}$ that is unresponsive to diazoxide poses a major therapeutic challenge. While subtotal pancreatectomy remains the procedure of choice following failure of medical therapy, the surgery is not completely curative and may still be associated with unsatisfactory glycemic control. Fluorine-18-dihydrophenylalanine positron emission tomography was not performed in our patient before surgery, since it was not available in children in China at the time, but based on the increased number of islets and enlarged volume of partial regional islets reported histopathologically, as well as the recurrent severe hypoglycemia after subtotal pancreatectomy, the patient likely had a diffuse $\mathrm{CHI}$. The patient has a heterozygous mutation in ABCC8. Definitely, most dominant acting monoallelic potassium channel ATP gene mutations cause mild diazoxide responsive CHI. However, Saint-Martin et al (16) reported that some dominant $A B C C 8$ mutations are responsible for a subset of diffuse, diazoxide-unresponsive forms of $\mathrm{CHI}$. The mechanism in these cases is unclear and needs further study. After pancreatectomy, the total amount of nasogastric and parenteral fluids had reached maximum, and it was also very difficult to establish a central venous line. Therefore, there was a need for an alternative treatment, minimizing the requirement for repeat pancreatectomy, and the burden of demanding medical and nutritional intervention in our patient.

Sirolimus has been reported as a treatment option for unresponsive $\mathrm{CHI}(10,11,12,13)$. No major adverse reactions were observed during follow-up period in these case reports, though a recent study in two large centers showed that mTOR inhibition achieved euglycemia, fasting tolerance and reduced medical therapy in only $30 \%$ of patients and more adverse events were observed (17). mTOR is a serine and threonine protein kinase that integrates signals from mitogens and nutrients, glucose and amino acids, to regulate cellular growth and proliferation (18). The mechanism of mTOR inhibitors in CHI has not been fully delineated. Hyperplasia of $\beta$-cells has been proposed to be involved in the trans-differentiation of mature acinar and ductal elements of exocrine pancreas into insulin-secreting cells, which is possibly mediated by the constitutive activation of the mTOR pathway (19). mTOR inhibition may also affect the number of insulin receptors that are present in pancreatic $\beta$-cells, which would reduce insulin production (20). Sirolimus has been used in vitro to induce fulminant diabetes by promoting insulin resistance and reducing $\beta$-cell mass through apoptosis induction $(21,22)$. Furthermore, long-term management with sirolimus was found to cause glucose intolerance by up-regulating hepatic gluconeogenesis (23). It is postulated that the mechanism of mTOR inhibition is also reduced during islet cell proliferation $(11,12)$. This was recently confirmed by genomic datasets implicating the insulin-like growth factor $1 / \mathrm{mTOR} / \mathrm{Akt}$ pathway in the pathophysiology of $\mathrm{CHI}$ (9). Another study has shown that mTOR pathways are not downregulated in keeping with non-responsiveness to sirolimus and the observation that proliferation remains high after treatment with sirolimus (17). 
The reported adverse effects of sirolimus treatment include stomatitis, fatigue, immunosuppression, increased risk of infections, renal function abnormalities, hyperlipidemia, and pneumonitis $(22,24)$, which are reversible with dose reduction. Mild elevation of triglycerides was observed in our patient. Sirolimus appears to be well tolerated in children post renal transplant, even when initiated at higher doses (6 $\mathrm{mg} / \mathrm{m}^{2} /$ day) and thereafter adjusted to achieve target trough levels in the range of $10-20 \mathrm{ng} / \mathrm{mL}$ (25). These studies suggest a reasonable safety profile for sirolimus, but the long-term safety remains unknown in younger children, particularly in neonates.

\section{Conclusion}

In conclusion, sirolimus was a well-tolerated treatment in our patient with $\mathrm{CHI}$ who otherwise would have required second surgery, and no major adverse events were observed during the period of 10 months of treatment. Sirolimus may be a feasible option for selected $\mathrm{CHI}$ patients with no contraindication, either before surgery or as an adjunctive therapy, although the mechanism and long-term adverse effects of such treatment require further study.

\section{Acknowledgment}

We thank Zhengzhou Kingmed Clinical Laboratory Center for their providing free genetic testing.

\section{Ethics}

Informed Consent: Informed consent was obtained from the parents of this patient.

Peer-review: Externally peer-reviewed.

\section{Authorship Contributions}

Concept: Yun Yan, Haiyan Wei, Design: Haiyan Wei, Yongxing Chen, Data Collection or Processing: Qiong Chen, Xiaohong Wang, Haihua Yang, Yingxian Zhang, Xiaojing Liu, Analysis or Interpretation: Yongxing Chen, Yun Yan, Literature Search: Yongxing Chen, Yun Yan, Writing: Qiong Chen, Yongxing Chen, Yun Yan, Haiyan Wei.

Financial Disclosure: The authors declared that this study received no financial support.

\section{References}

1. Senniappan S, Shanti B, James C, Hussain K. Hyperinsulinaemic hypoglycaemia: genetic mechanisms, diagnosis and management. J Inherit Metab Dis 2012;35:589-601. Epub 2012 Jan 10

2. Senniappan S, Arya VB, Hussain K. The molecular mechanisms, diagnosis and management of congenital hyperinsulinism. Indian J Endocrinol Metab 2013;17:19-30.
3. Arnoux JB, Verkarre V, Saint-Martin C, Montravers F, Brassier A, Valayannopoulos V, Brunelle F, Fournet JC, Robert JJ, Aigrain Y, Bellanné-Chantelot $\mathrm{C}$, de Lonlay P. Congenital hyperinsulinism: current trends in diagnosis and therapy. Orphanet J Rare Dis 2011;6:63.

4. Rahier J, Guiot Y, Sempoux C. Morphologic analysis of focal and diffuse forms of congenital hyperinsulinism. Semin Pediatr Surg 2011;20:3-12.

5. Rahier J, Guiot Y, Sempoux C. Persistent hyperinsulinaemic hypoglycaemia of infancy: a heterogeneous syndrome unrelated to nesidioblastosis. Arch Dis Child Fetal Neonatal Ed 2000;82:108-112.

6. Palladino AA, Stanley CA. A specialized team approach to diagnosis and medical versus surgical treatment of infants with congenital hyperinsulinism. Semin Pediatr Surg 2011;20:32-37.

7. Meissner T, Wendel U, Burgard P, Schaetzle S, Mayatepek E. Longterm follow-up of 114 patients with congenital hyperinsulinism. Eur J Endocrinol 2003;149:43-51.

8. Arya VB, Senniappan S, Demirbilek H, Alam S, Flanagan SE, Ellard $S$, Hussain K. Pancreatic endocrine and exocrine function in children following near-total pancreatectomy for diffuse congenital hyperinsulinism. PLos One 2014;9:e98054.

9. Senniappan S, Brown R, Hussain K. Genomic and morphoproteomic correlates implicate the IGF-1/mTOR/Akt pathway in the pathogenesis of diffuse congenital hyperinsulinism. Int J Clin Exp Pathol 2016;9:548562 .

10. Abraham MB, Shetty VB, Price G, Smith N, Bock Md, Siafarikas A, Resnick S, Whan E, Ellard S, Flanagan SE, Davis EA, Jones TW, Hussain K, Choong CS. Efficacy and safety of sirolimus in a neonate with persistent hypoglycaemia following near-total pancreatectomy for hyperinsulinaemic hypoglycaemia. J Pediatr Endocrinol Metab 2015;28:1391-1398.

11. Senniappan S, Alexandrescu S, Tatevian N, Shah P, Arya V, Flanagan S, Ellard S, Rampling D, Ashworth M, Brown RE, Hussain K. Sirolimus therapy in infants with severe hyperinsulinemic hypoglycemia. N Engl J Med 2014;370:1131-1137.

12. Méder Ü, Bokodi G, Balogh L, Körner A, Szabó M, Pruhova S, Szabó AJ. Severe Hyperinsulinemic Hypoglycemia in a Neonate: Response to Sirolimus Therapy. Pediatrics 2015;136:1369-1372.

13. Shah P, Arya VB, Flanagan SE, Morgan K, Ellard S, Senniappan S, Hussain K. Sirolimus therapy in a patient with severe hyperinsulinaemic hypoglycaemia due to a compound heterozygous ABCC8 gene mutation. J Pediatr Endocrinol Metab 2015;28:695-699.

14. Henwood MJ, Kelly A, Macmullen C, Bhatia P, Ganguly A, Thornton PS, Stanley CA. Genotype-phenotype correlations in children with congenital hyperinsulinism due to recessive mutations of the adenosine triphosphate-sensitive potassium channel genes. J Clin Endocrinol Metab 2005;90:789-794.

15. Richards S, Aziz N, Bale S, Bick D, Das S, Gastier-Foster J, Grody WW, Hegde M, Lyon E, Spector E, Voelkerding K, Rehm HL; ACMG Laboratory Quality Assurance Committee. Standards and guidelines for the interpretation of sequence variants: a joint consensus recommendation of the American College of Medical Genetics and Genomics and the Association for Molecular Pathology. Genet Med 2015;17:405-423. Epub 2015 Mar 5

16. Saint-Martin C, Zhou Q, Martin GM, Vaury C, Leroy G, Arnoux JB, de Lonlay P, Shyng SL, Bellanné-Chantelot C. Monoallelic ABCC8 mutations are a common cause of diazoxide-unresponsive diffuse form of congenital hyperinsulinism. Clin Genet 2015;87:448-454. Epub 2014 Jun 6

17. Szymanowski M, Estebanez MS, Padidela R, Han B, Mosinska K, Stevens A, Damaj L, Pihan-Le Bars F, Lascouts E, Reynaud R, Ferreira C, Bansept C, de Lonlay P, Saint-Martin C, Dunne MJ, Banerjee I, Arnoux JB. mTOR Inhibitors for the Treatment of Severe Congenital 
Hyperinsulinism: Perspectives on Limited Therapeutic Success. J Clin Endocrinol Metab 2016;101:4719-4729. Epub 2016 Oct 3

18. Kwon G, Marshall CA, Pappan KL, Remedi MS, McDaniel ML. Signaling elements involved in the metabolic regulation of mTOR by nutrients, incretins, and growth factors in islets. Diabetes 2004;53(Suppl 3):225232.

19. Alexandrescu S, Tatevian N, Olutoye O, Brown RE. Persistent hyperinsulinemic hypoglycemia of infancy: constitutive activation of the mTOR pathway with associated exocrine-islet transdifferentiation and therapeutic implications. Int J Clin Exp Pathol 2010;3:691-705.

20. Leibiger IB, Leibiger B, Moede T, Berggren PO. Exocytosis of insulin promotes insulin gene transcription via the insulin receptor/PI-3 kinase/p70 s6 kinase and CaM kinase pathways. Mol Cell 1998;1:933938.

21. Yang SB, Lee HY, Young DM, Tien AC, Rowson-Baldwin A, Shu YY, Jan YN, Jan LY. Rapamycin induces glucose intolerance in mice by reducing islet mass, insulin content, and insulin sensitivity. J Mol Med (Berl) 2012;90:575-585. Epub 2011 Nov 22
22. Sankhala K, Mita A, Kelly K, Mahalingam D, Giles F, Mita M. The emerging safety profile of mTOR inhibitors, a novel class of anticancer agents. Target Oncol 2009;4:135-142. Epub 2009 Apr 21

23. Houde VP, Brûlé S, Festuccia WT, Blanchard PG, Bellmann K, Deshaies $\mathrm{Y}$, Marette A. Chronic rapamycin treatment causes glucose intolerance and hyperlipidemia by upregulating hepatic gluconeogenesis and impairing lipid deposition in adipose tissue. Diabetes 2010;59:13381348. Epub 2010 Mar 18

24. Thomas NJ, Brooke AM, Besser GM. Long-term maintenance of normoglycaemia using everolimus in a patient with disseminated insulinoma and severe hypoglycaemia. Clin Endocrinol (Oxf) 2013;78:799-800.

25. Schachter AD, Benfield MR, Wyatt RJ, Grimm PC, Fennell RS, Herrin JT, Lirenman DS, McDonald RA, Munoz-Arizpe R, Harmon WE. Sirolimus pharmacokinetics in pediatric renal transplant recipients receiving calcineurin inhibitor co-therapy. Pediatr Transplant 2006;10:914-919. 